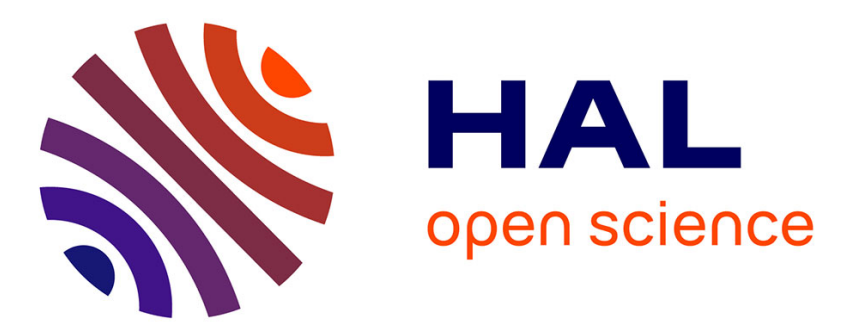

\title{
A Fictitious Domain Approach Based on a Viscosity Penalty Method to Simulate Wave/Structure Interaction
}

Benoît Ducassou, Jonathan Nuñez, Marcela Cruchaga, Stéphane Abadie

\section{To cite this version:}

Benoît Ducassou, Jonathan Nuñez, Marcela Cruchaga, Stéphane Abadie. A Fictitious Domain Approach Based on a Viscosity Penalty Method to Simulate Wave/Structure Interaction. Journal of Hydraulic Research, 2017, 55 (6), pp.847-862. 10.1080/00221686.2017.1289257 . hal-02153319

\section{HAL Id: hal-02153319 \\ https: / hal-univ-pau.archives-ouvertes.fr/hal-02153319}

Submitted on 10 Jun 2021

HAL is a multi-disciplinary open access archive for the deposit and dissemination of scientific research documents, whether they are published or not. The documents may come from teaching and research institutions in France or abroad, or from public or private research centers.
L'archive ouverte pluridisciplinaire HAL, est destinée au dépôt et à la diffusion de documents scientifiques de niveau recherche, publiés ou non, émanant des établissements d'enseignement et de recherche français ou étrangers, des laboratoires publics ou privés. 
To appear in the Journal of Hydraulic Research

Vol. 00, No. 00, Month 20XX, 1-17

Research paper

\title{
A Fictitious Domain approach based on a viscosity penalty method to simulate wave/structure interaction
}

B. DUCASSOU, Doctor, UNIV. PAU \& PAYS ADOUR, Laboratoire Sciences de l'Ingénieur Appliquées á la Mécanique et au Génie Electrique, EA4581, Allée du Parc Montaury, 64600 Anglet, France

Email: benoit.ducassou@univ-pau.fr

J. NUÑEZ, MSc Student, Universidad de Santiago de Chile (USACH), Av. Bdo. O'Higgins 3363, Estación Central Santiago de Chile, Chile

Email: jonathan.nuneza@usach.cl

M. CRUCHAGA, Professor, Universidad de Santiago de Chile (USACH), Av. Bdo. O'Higgins 3363, Estación Central Santiago de Chile, Chile

Email: marcela.cruchaga@usach.cl

S. ABADIE, Professor, UNIV. PAU \& PAYS ADOUR, Laboratoire Sciences de l'Ingénieur Appliquées á la Mécanique et au Génie Electrique, EA4581, Allée du Parc Montaury, 64600 Anglet, France

Email: stephane.abadie@univ-pau.fr

Running Head : A viscosity penalty method for wave/structure interaction

\begin{abstract}
This work presents an original numerical model for a free surface flow interacting with a spring-block system. The formulation is based on the fictitious domain approach and a penalty method on viscosity to describe the rigid solid motion. The incompressible Navier-Stokes equations are solved in the whole domain and the free surface and the body contour are captured using a Volume of Fluid method. To describe the rigid body motion, a single degree of freedom model, able to represent translation or rotation, is embedded in the code. The discrete equations are written in a well known finite volume framework over Cartesian grids. In such a context, the external spring and damping forces are represented as body forces in the solid region. The proposed strategy is tested in a sloshing damping system. The numerical results are compared with experimental data obtained within the present study. Finally, the method is used to simulate a wave energy converter system as an illustration of a rotational case.
\end{abstract}

Keywords: Computational methods in hydro-environment research and fluid dynamics; flow-structure interactions: flow control: hydraulics of renewable energy systems: laboratory studies; wave-structure interactions.

\section{Introduction}

Free surface flows interacting with moving rigid bodies have been widely reported in the literature due to their extensive use in many hydraulic applications. Wave interaction with coastal defence elements (e.g. Rogers, Dalrymple, \& Stansby, 2010) or floating breakwaters (e.g. Huang, He, \& Zhang, 2014; Koftis, Prinos, \& Koutandos, 2006), wave action on ships or off-shore platforms (e.g. Zhao, Ye, Fu, \& Cao, 2014), wave energy recovery systems (e.g. Renzi, Abdolali, Bellotti, \& Dias, 2014; Y.-H. Yu \& Li, 2013), or conversely, generation of waves by rock fall in water reservoirs (e.g. Abadie, Morichon, Grilli, \& Glockner, 2010), all require the description of liquid-gas interfaces interacting with moving bodies. These types of problems also often involve external forces which 
can act on the solid, such as mooring forces on floating bodies, power take-off mechanisms in wave energy converters, or a friction force as at the interface between a breakwater caisson and the underlying rubble mound.

The Fluid Solid Interaction (FSI) involves the coupling between two different phenomena: the fluid flow usually expressed in the Eulerian reference frame and the structure deformation for which, the Lagrangian frame is more adapted. Many numerical methods try to solve this intrinsic difficulty. Among these, the Arbitrary Lagrangian-Eulerian method (ALE) (Donea, Giuliani, \& Halleux, 1982), is likely the most often used, and consists in deforming the fluid domain from a reference configuration to follow the fluid/solid interface. This method is for instance used in Kassiotis, Ibrahimbegovic, and Matthies (2010) to compute the impact of a free surface flow generated by a dam break on a flexible obstacle.

When the solid is rigid, the study is somehow simplified, but appropriate numerical methods are still required. Numerical methods can be grouped in two classes: moving or fixed grid methods. The Smoothed Particle Hydrodynamics approach (SPH) (Gingold \& Monaghan, 1977) is a meshless method where the unknowns are computed at points that move during the analysis. It was recently adapted to FSI by Groenenboom and Cartwright (2010). An example of the application of this method is reported in Altomare et al. (2014) for the simulation of the interaction of waves with sea breakwater armor blocks. The Boundary Element Method (BEM) is also belongs to Lagrangian methods. Here, the problem is reduced to a discretization over the boundary of the domain and the flow is assumed to be potential (Borgarino, Babarit, \& Ferrant, 2012). Methods with a fixed mesh include the family of fictitious domain method, where we find the Immersed Boundary Method (IBM) (Peskin, 1972, 2002), the Distribute Lagrangian multiplier (DLM) (Glowinski, Pan, Hesla, Joseph, \& Periaux, 2001), penalty methods (Angot, Bruneau, \& Fabrie, 1999) among others. These methods superpose the body on a fixed fluid domain generating a fictitious fluid domain where the solid is. It is necessary to impose conditions on the fictitious fluid domain to ensure non-penetration of fluid through the solid boundary. The IBM method consists in moving the solid wall and imposing a jump of the stress at the interface. This condition is achieved by imposing additional forces on the fluid formulation in the vicinity of the solid boundary. The DLM method principle is to fill the solid areas with a virtual fluid, to relax the rigid body motion condition of the rigid body, and then impose the rigid body motion by Lagrange multipliers in the variational formulation of the fluid (Glowinski et al., 2001). This approach was later extended to the interaction with a deformable body (Yu, 2005). Among the penalty methods we find the Euler-Lagrangian coupling method where a solid Lagrangian mesh is superimposed onto a fixed fluid Eulerian mesh. The principle is to penalize the velocity difference between the solid interface and the fluid (Sarthou, Vincent, Caltagirone, \& Angot, 2008). Non-Lagrange multiplier fictitious domain methods can also be used to solve FSI problems as presented in Veeramani, Minev, and Nandakumar (2007).

A local penalization of the fluid viscosity can also be used to reproduce quasi solid behaviour. This approach seems to appear first in Ritz and Caltagirone (1999) in the context of fluid particle interactions. The method was further developed in Caltagirone and Vincent (2001), Randrianarivelo, Pianet, Vincent, and Caltagirone (2005), and Vincent, Randrianarivelo, Pianet, and Caltagirone (2007) by separating the different contributions of the deformation rate tensor. Penalization on viscosity can also be found in Cruchaga, Celentano, and Tezduyar (2007), where a finite element numerical model based on the mixed interface-tracking/interface-capturing technique (MITICT) for flows with fluid-solid and fluid-fluid interfaces is used to simulate a nylon sphere falling in a fluid-filled tube. In the MITICT, fluid / solid interfaces are accurately tracked with a moving Lagrangian technique, and fluid / fluid interfaces are treated with an interface-capturing technique as they are too complex and unsteady to track (Cruchaga, Löhner, \& Celentano, 2012).

The viscosity penalization approach is also followed in the present study. In this paper, we present a finite volume method including the Volume of Fluid (VOF) model reported in Abadie et al. (2010) to deal with an immersed rigid solid in motion subjected to external forces. To this end, a formulation is proposed including the following aspects: two VOF are implemented to track 
the fluid-fluid and fluid-solid interfaces, a penalty model on the viscosity is used to describe solid regions, and an algorithm to describe external forces acting on the fluid-structure system is included. The resulting methodology is able to solve the fluid interaction with rigid bodies considering a free surface flow behaviour like those presented in sloshing dampers or marine energy systems.

Compared to previous works, the novelty includes:

- the application of this penalty method generally restrained to fluid particle interactions (e.g., Ritz \& Caltagirone, 1999) to hydraulic engineering systems,

- the definition of a rigidity criterion and a simple method to impose kinematic constraints on the solid such as the definition of a rotation center,

- a practical approach to define external forces acting on the solid body,

- finally, novel experiments allowing to assess the numerical behaviour of the proposed technique and its potential to solve an immersed spring-block system.

The outline of the paper is as follows: Section 2 reports the governing equations and the numerical method used to solve them. The proposed penalty method based on body forces and a velocity constraint is also detailed in Section 2. Section 3 presents the experiment that has been conducted to validate the numerical method in translation. The comparison between the experimental data and the simulation results are summarized in the same section. In Section 4, an example of application of the method to a rotational system (i.e. the wave roller energy converter) is given. Finally, perspectives and conclusions are drawn in Section 5.

\section{Model Presentation}

\subsection{Governing Equations}

The problem to be analysed is sketched in Fig. 1. It involves two fluids (i.e. water $\Omega_{F 1}$ and air $\Omega_{F 2}$ ) and a solid body $\Omega_{S}$. The following incompressible Navier-Stokes equations for a Newtonian fluid describe the flow motion in the fluid domain (i.e., $\Omega_{F}=\Omega_{F 1} \cup \Omega_{F 2}$ ):

$$
\begin{gathered}
\nabla \cdot \boldsymbol{v}=0 \\
\rho\left(\frac{\partial \boldsymbol{v}}{\partial t}+(\boldsymbol{v} \cdot \nabla) \boldsymbol{v}\right)+\nabla p-\nabla \cdot\left[\mu\left(\boldsymbol{\nabla} \boldsymbol{v}+\nabla^{t} \boldsymbol{v}\right)\right]=\rho \boldsymbol{b}
\end{gathered}
$$

where $\rho$ and $\mu$ are respectively fluid density and viscosity, both spatially variable and symbol $\boldsymbol{b}$ represents an acceleration (typically the gravity acceleration) leading to the body force. This system of equations is completed with appropriate initial and boundary conditions. Moreover, the interface between $\Omega_{F 1}$ and $\Omega_{F 2}$, named $\Gamma_{F}$, needs to be described including kinematic and dynamic conditions at the interface. Here continuity of velocity and stress through $\Gamma_{F}$ is assumed (i.e., in the present work no surface tension is considered).

The rigid solid motion of the body $\Omega_{S}$ is defined by Newton's laws, written in terms of displacement of its centroid $\boldsymbol{\delta}$ and its angular rotation $\theta$ in two dimensions by :

$$
\begin{aligned}
m \ddot{\boldsymbol{\delta}} & =\boldsymbol{F}_{\text {ext }} \\
I \ddot{\theta} & =T_{\text {ext }}
\end{aligned}
$$

where $m$ is the rigid body mass, $\boldsymbol{F}_{\text {ext }}$ is the global external force acting on $\Omega_{S}$ (including gravity and hydrodynamic force),$I$ is the solid inertia moment and $T_{e x t}$ the hydrodynamic torque acting 
on the solid center of mass. The continuity of velocity and stress should also be ensured through the solid contour $\Gamma_{S}$.

The fluid-solid interaction basically requires:

1. solving equations 1 and 2 over the $\Omega_{F}$ domain including free surface motion,

2. computing $\boldsymbol{F}$ and $\boldsymbol{T}$ acting on the rigid body $\Omega_{S}$,

3. solving equations 3 and 4 and moving $\Omega_{S}$ accordingly,

4. recomputing the flow equations (step 1 ) in the updated $\Omega_{F}$ domain.

In the present work, an alternative formulation to the described fluid-solid interaction problems for rigid bodies is used. Equations 1 and 2 are solved in the whole domain $\Omega=\Omega_{F} \cup \Omega_{S}$. The viscosity inside the $\Omega_{S}$ region is taken large enough to enforce rigid conditions and it acts as a penalty factor of the strain-rate tensor. In addition, the interface evolution for $\Gamma_{F}$ and $\Gamma_{S}$ needs to be determined. To this end, the motion of each surface is described by:

$$
\frac{\partial \Phi_{F, S}}{\partial t}+\boldsymbol{v} \cdot \nabla \Phi_{F, S}=0
$$

where $\Phi_{F, S}(x, y, z)$ is a phase function corresponding to $\Gamma_{F}$ and $\Gamma_{S}$, respectively.

\subsection{Numerical method for NS equations}

The NS equations are discretized on a fixed Cartesian grid using a finite volume formulation. Following Patankar (1980), the finite volume formulation is solved using a staggered mesh known as the Marker And Cells (MAC) method from Harlow, Welch, et al. (1965). The coupling between velocity and pressure is solved using the Augmented Lagrangian method described by Fortin and Glowinski (1982). This is a minimization method under the constraint of the continuity equation, where the pressure, which is decoupled from the velocity, appears as a Lagrange multiplier. The incompressibility constraint is directly introduced into the equation of motion as a penalty term $r_{u} \nabla(\nabla \cdot v)$, that couples the velocity components. At each time step $n$ and at iteration $k$, the system is written as:

$$
\begin{gathered}
\rho^{n}\left(\frac{\boldsymbol{v}^{n, k+1}}{\triangle t^{n}}+\left(\boldsymbol{v}^{n, k} \nabla\right) \boldsymbol{v}^{n, k+1}\right)-\rho^{n} \boldsymbol{b} \\
-\nabla p^{n, k} \\
-\nabla\left[\mu^{n}\left(\nabla \boldsymbol{v}^{n, k+1}+\nabla^{t} \boldsymbol{v}^{n, k+1}\right)\right]-r_{u} \nabla\left(\nabla \cdot \boldsymbol{v}^{n, k+1}\right)=\rho^{n} \frac{\boldsymbol{v}^{n}}{\triangle t^{n}} \\
p^{n, k+1}=p^{n, k}-r_{p} \nabla \cdot \boldsymbol{v}^{n, k+1},
\end{gathered}
$$

where $r_{u}$ and $r_{p}$ are convergence parameters set in the present work as 1 . The advantage of such a formulation is the explicit calculation of the pressure. It uses only the pressure in the previous temporal iteration and the divergence of velocity. When turbulence effects need to be included, the viscosity $\mu$ in equation (6) is usually modified by using adequate turbulence models. For completeness, in the present version of the developed computational code, the viscosity $\mu$ is the sum of the molecular viscosity and an additional viscosity calculated by the Large Eddy Simulation (LES) model reported by Sagaut, Troff, Lê, and Loc (1996). This additional viscosity was found to help to stabilize computations for the most non linear sloshing cases studied in Ducassou (2016). In the cases presented in this paper, no turbulence model is required. as the turbulence is weak and does not significantly influence the flow. Nevertheless, the additional viscosity was kept to ensure numerical stabilization in cases with the most violent block motions avoiding to reduce mesh size 
and time step. Finally, note that the LES model is also adapted to the FSI approach presented in this paper. Therefore, it could be employed to study turbulence in more detail for future 3D applications where sufficiently resolved meshes need to be chosen.

\subsection{Penalty method}

To model a mobile solid, the use of a penalty model on the viscosity (Angot et al., 1999; Ritz \& Caltagirone, 1999) is proposed. This method allows to stiffen certain areas of the fluid field. In fact, a very high viscosity value is used in the solid region enforcing the fluid in such a region to mimic the rigid body behaviour. The viscosity acts as a penalization parameter, and it promotes nearly zero strain-rates in the solid domain (i.e., non-deformations are obtained) while still allowing solid motion. Indeed, when a very large viscosity is used in a certain area of the domain, the viscous stress tensor term becomes predominant in the discretized momentum equation 6 in this area. Then, the strain-rate tensor has to be close to zero when numerical convergence is achieved. Practically, the penalized viscosity has to be within a certain range to ensure a sufficient non-deformation criterion while keeping convergence of the algorithm.

Therefore, inside the solid body contour only a zero deformation criterion is in fact solved using this method. So following this constraint, the model gives a constant velocity inside the body contour. But the value of this velocity actually depends on the hydrodynamics forces which act on the body interface. In the first liquid cell (i.e., containing zero body volume fraction) just close to the body, the classic Navier-Stokes equations are solved (i.e., without the viscosity penalty), thus providing physical pressure and velocities. The velocity inside the rigid body is a global result from these forcings. Actually, the equilibrium is solved implicitly at once through the Augmented Lagrangian procedure and the inversion of the linear system, and the result is that the equations system $(6,7)$ will be equivalent to the system $(3,4)$ in the solid area.

\section{Rigidity criterion}

In order to quantitatively control the non-deformation of the stiffened fluid region, the following approach is proposed. First, a coefficient of local deformation $C_{d e f}$ is computed at each mesh cell $i, j$ (here written in $2 \mathrm{D}$ ):

$$
C_{d e f_{i, j}}=\sqrt{\varepsilon_{I_{i, j}}^{2}+\varepsilon_{I I_{i, j}}^{2}}
$$

where $\varepsilon_{I}$ and $\varepsilon_{I I}$ are the strain rate deformation tensor components for the two main axes. If this coefficient is very low, it means that the particle does not become locally distorted according to its two main axes. At every time step, the maximum of $C_{d e f_{i, j}}$ is computed over the solid volume as $C_{d e f}(t)=\max \left[C_{d e f_{i, j}}\right]$ at each time. Finally, an accumulated maximum deformation $C_{t o t}$ is assessed by summing $C_{t o t}$ over the simulation time $Y$ as:

$$
C_{t o t}=\int_{Y} C_{d e f}(t) \mathrm{d} t=\sum_{i} C_{d e f}\left(t_{i}\right) \mathrm{d} t_{i}
$$

In the simulations performed in this paper, the $C_{t o t}<0.1 \%$ criterion was chosen to achieve an appropriate description of the rigid body motion. Practically, this criterion is ensured with a penalized viscosity of $o\left(10^{7}\right) \mathrm{Pa}$ s.

\section{Kinematic constraint}

Kinematic constraints need to be considered when dealing with hydraulic engineering systems. For instance, to promote purely translational or rotational (e.g., the wave energy converter described 
in 4) rigid body motions. In the framework of this method, this can be very simply achieved by penalizing the velocity within the solid fluid area. For instance, to impose horizontal translation, the vertical velocity is fixed to zero at two successive grid points of the velocity mesh (horizontal velocity is fixed to obtain a vertical translation and so on). Technically, this is achieved by using a supplementary penalty term in the resolution matrix. To force rotational motion around an axis, the center of rotation is taken at a pressure node, and the four velocity neighbours are penalized within the resolution matrix and fixed at zero.

\section{External forces}

External forces acting on a solid body in interaction with a fluid are also often involved in hydraulic applications. Here the method is described for spring and damper forces, but it can be generalized to other forces such as Coulomb friction force, magnetic forces, etc. The idea is to see that body forces and point forces are equivalent as soon as rigid motion is considered. Therefore, in the present work, the spring and damper forces are taken in consideration as supplementary accelerations $\left(\boldsymbol{A}_{\text {ext }}\right)$ superimposed onto gravity $(\boldsymbol{g})$, resulting in the following expressions:

$$
\begin{gathered}
\boldsymbol{b}=\boldsymbol{g}+\boldsymbol{A}_{\text {ext }} \\
\boldsymbol{A}_{\text {ext }}=\frac{1}{\rho_{S} \Omega_{S}}\left[\begin{array}{c}
-\mathrm{k}_{\mathrm{x}} \delta_{x}-\mathrm{c}_{\mathrm{x}} \frac{\mathrm{d} \delta_{x}}{\mathrm{~d} t} \\
-\mathrm{k}_{\mathrm{y}} \delta_{y}-\mathrm{c}_{\mathrm{y}} \frac{\mathrm{d} \delta_{y}}{\mathrm{~d} t}
\end{array}\right]+\frac{1}{\rho_{S} \Omega_{S}}\left[\begin{array}{l}
\frac{-\mathrm{k}_{\mathrm{r}} \theta \sin \left(\theta_{M}\right)}{r_{M}}-\frac{\mathrm{c}_{\mathrm{r}} \dot{\theta} \sin \left(\theta_{M}\right)}{r_{M}} \\
\frac{-\mathrm{k}_{\mathrm{r}} \theta \cos \left(\theta_{M}\right)}{r_{M}}-\frac{\mathrm{c}_{\mathrm{r}} \dot{\theta} \cos \left(\theta_{M}\right)}{r_{M}}
\end{array}\right]
\end{gathered}
$$

where $\mathrm{k}_{\mathrm{x}}$ and $\mathrm{k}_{\mathrm{y}}$ are the spring constants related to each reference axis; $\mathrm{k}_{\mathrm{r}}$ is the rotational spring constant; $\mathrm{c}_{\mathrm{x}}$ and $\mathrm{c}_{\mathrm{y}}$ are the damping coefficients; $\mathrm{c}_{\mathrm{r}}$ is the rotational damping coefficient; $r_{M}$ and $\theta_{M}$ are the polar coordinates of point $\mathrm{M}$ relative to the rotation center. To add acceleration $\boldsymbol{A}_{\text {ext }}$ in the discrete scheme, gravity is modified only in the grid points where the external forces act, i.e., into the rigid solid domain. The solid velocity components along the $x$ and $y$ axes are expressed as $\mathrm{d} \delta_{x} / \mathrm{d} t$ and $\mathrm{d} \delta_{y} / \mathrm{d} t$, respectively. Numerically, these terms are calculated by averaging out fluid velocity over the rigid body area as follows:

$$
\frac{\mathrm{d} \delta_{x}}{\mathrm{~d} t}=\frac{\sum_{1}^{n_{M}} u_{M}}{n_{M}} \quad \text { and } \quad \frac{\mathrm{d} \delta_{y}}{\mathrm{~d} t}=\frac{\sum_{1}^{n_{M}} v_{M}}{n_{M}}
$$

where $n_{M}$ is the number of velocity grid points in the solid, $\delta_{x}$ and $\delta_{y}$ are solid horizontal and vertical displacements with respect to its equilibrium position, $u_{M}$ and $v_{M}$ are the components of the velocity at point $\mathrm{M}$ along the $x$ and $y$ axes respectively. Numerically these terms are calculated at each time step $n$ by:

$$
\delta_{x}=\delta_{x_{\text {init }}}+\left.\sum_{i=1}^{n} \frac{\mathrm{d} \delta_{x}}{\mathrm{~d} t}\right|_{i} \triangle t_{i} \quad \text { and } \quad \delta_{y}=\delta_{y_{\text {init }}}+\left.\sum_{i=1}^{n} \frac{\mathrm{d} \delta_{\mathbf{y}}}{\mathrm{d} t}\right|_{i} \triangle t_{i}
$$

The solid angular velocity in the plane $\left(C_{r}, \boldsymbol{x}, \boldsymbol{y}\right)$ where $C_{r}$ is the rotation center, is computed as:

$$
\dot{\theta}=\frac{\sum_{n_{M}} \frac{\sqrt{u_{M}^{2}+v_{M}^{2}}}{r_{M}}}{n_{M}}
$$


The angular deviation $\theta$ of the solid from its equilibrium position in the plane $\left(C_{r}, \boldsymbol{x}, \boldsymbol{y}\right)$ :

$$
\theta=\theta_{\text {init }}+\sum_{i=1}^{n} \dot{\theta}_{i} \triangle t_{i}
$$

\subsection{Interface tracking and transport}

Equation 5 is solved using a Volume of Fluid (VOF) technique as described by Hirt and Nichols (1981), which describes the volume fraction occupied by one of the fluids (i.e., water) in a cell. The properties (density and viscosity) are distributed according to a mixing law based on the volume fraction at each cell. The Piecewise Linear Interface Calculation (PLIC) method is employed to recover the interface location from the discontinuous volume fraction distribution. The algorithm to advance the interface position in time reads:

- Identification of mixed cells (i.e., $0<\Phi_{F, S}<1$ ).

- In each mixed cell, calculation of the interface normal direction using a finite difference approximation on nine points.

- Piecewise linear interface reconstruction, knowing the normal direction in each cell and the volume fraction.

- Lagrangian advection of the interface segments using a linear interpolation of velocities calculated on the staggered mesh.

- Calculation of the new volume fraction distribution.

To ensure the stability of the PLIC method, a sufficient condition is that the volume fraction does not move more than half of a cell during a time step as was suggested by Abadie, Caltagirone, and Watremez (1998).

\subsection{Mesh size convergence analysis}

In the framework of this work, the simulation of a sphere settling in an enclosure at $\mathrm{R}=11.6$ reported by Ten Cate, Nieuwstad, Derksen, and Van den Akker (2002) has been performed to assess mesh size and time step convergence analysis. The material properties, corresponding to a Nylon bearing and a silicon oil, are $\rho_{\text {solid }}=1120 \mathrm{~kg} \mathrm{~m}^{-3}, \rho_{\text {liquid }}=962 \mathrm{~kg} \mathrm{~m}^{-3}$ and $\mu_{\text {liquid }}=0.113$ $\mathrm{Pa}$ s, respectively. The diameter of the sphere is $d=15 \mathrm{~mm}$ and the tube has a diameter $D=100$ $\mathrm{mm}$ with a height $L=160 \mathrm{~mm}$. The initial position of the sphere is $H=120 \mathrm{~mm}$ and $h_{x}$ and $h_{y}$ are the characteristic grid sizes for the $\mathrm{x}$ and $\mathrm{y}$ directions, respectively (uniform grids are used in this analysis, i.e. $h_{x}=h_{y}$, see Fig. 2a for details).

To verify the numerical behaviour of the penalty method, the evolution of the accumulated maximum deformation computed from equation 9 is reported in Fig. 2b, showing practically negligible deformations values. Figure $2 \mathrm{c}$ presents the time evolution for the sphere's velocity computed in the present work using different grids characterized by $\beta=d / h_{y}$. Mesh refinement ensures the convergence of the simulation results with the experimental data. In such a figure, the results reported by Cruchaga, Muñoz, and Celentano (2008) using a finite element formulation are also included to check the numerical behaviour of the present technique. Finally, the average CPU time for this simulation is $0.0007 \mathrm{~s}$ per cell and time step on an Intel $囚 \mathrm{E} 5-2650 \mathrm{Lv} 2$ processor. 


\section{Experimental work and validation of the numerical method in the translational case : the sloshing damper case}

\subsection{Description of the experiment}

This section introduces the experiments developed in the framework of the present work with the aim to obtain valuable data to validate the numerical formulation in a translational motion. The case studied could also be an original idea of sloshing damper. The setup of the experiment is presented in Fig. 3. A spring-block system put into an acrylic tank is attached to the lateral walls of the tank. The block is mounted on wheels so that bottom friction can be neglected. The tank is mounted on a shake table able to produce controlled amplitude motions $A$ and angular frequency $\omega=2 \pi f$. The dimensions of the different components of the system are presented in Table 1 . The tank is filled with water up to a level $h$. Two cases were studied:

1. Spring-block system only, i.e. the tank is empty,

2. Spring-block system immersed, i.e., the tank is filled up to different levels and the free-surface evolves with time. In the present work, only the experiments with a water level at rest $h=400$ $\mathrm{mm}$ are reported.

In addition, the experimental case of a spring-block system confined (i.e. the tank is fully filled with water, with no-free surface is present), was also conducted. Practically no block motion was detected, hence the experimental and numerical results obtained for this case are not shown (Ducassou, 2016; Nuñez, 2015).

The experiments are carried out with an imposed motion of amplitude $A=5 \mathrm{~mm}$ using different imposed frequencies $f$ ranging from $1 \mathrm{~Hz}$ to $6 \mathrm{~Hz}$. The experiments are video recorded using a high speed camera with an image quality of $800 x 600$ pixels at 200 or $500 \mathrm{fps}$. The motions of the block and the shake table are obtained from such videos using a motion capturing technique. The error in the positions is estimated as $\pm 0.25 \mathrm{~mm}$ based on pixel size. From such data, the amplitudes of the block motion and the phases related to the table position are calculated. Moreover, the free surface evolution is captured by ultrasonic sensors located at $25 \mathrm{~mm}$ from the walls of the tank (labelled S1, S2, S3, and S4; see Fig. 3(b)). Sensor S5 is used to register the motion of the tank, which coincides with the shake table motion, but the data from S5 helps to synchronize the signals. The error in the sensor measurements is $\pm 0.5 \mathrm{~mm}$. During 2D behaviour of the free surface evolution, coincident signals at S1 and S2 or S3 and S4 are reported, as illustrated in Fig. 4(a) at an imposed frequency of $1.25 \mathrm{~Hz}$. Meanwhile, differences in the signals registered by S1 and S2 or by S3 and $\mathrm{S} 4$ are expected only when 3D effects of the free surface evolution become significant (e.g. the free surface response obtained at imposed frequency of $1.9 \mathrm{~Hz}$, see Fig. 4(b)). From the experiments, 2D free surface behaviour was found at imposed frequencies of $0.5 \mathrm{~Hz}, 0.75 \mathrm{~Hz}, 1.0 \mathrm{~Hz}, 1.25 \mathrm{~Hz}, 1.6$ $\mathrm{Hz}, 1.7 \mathrm{~Hz}$, and $1.8 \mathrm{~Hz}$. The free surface evolution clearly shows 3D effects at imposed frequencies of $1.9 \mathrm{~Hz}, 2.0 \mathrm{~Hz}, 2.2 \mathrm{~Hz}$, and $2.4 \mathrm{~Hz}$. The experiments report that free surface 3D effects evolve near resonance frequencies. Due to the experimental configuration, 3D effects are only detected by the free surface evolution, while the block motion is constrained to be one-directional. The experimental measurements obtained during the time-periodic regime (i.e. the steady state forced vibration) are reported in the next section together with the computed numerical responses.

\section{Mechanical characterization of the spring-block system}

The mechanical parameters (spring rigidity $k$ and system damping $c$ ) need to be determined to model the problem. A least squares approximation can be used to adjust such parameters considering that the experimental response for case 1 must adjust the analytical spring-block solution. Neglecting air drag and added mass effect, the equation of motion of a spring-damper-block system 
subjected to imposed support motion is written as:

$$
m \ddot{x}_{\text {solid }}+c \dot{x}_{\text {solid }}+k x_{\text {solid }}=c \dot{x}_{\text {table }}+k x_{\text {table }}
$$

In this equation $x_{\text {solid }}(t)$ and $x_{\text {table }}(t)$, represent the motion of the block and the motion of the table, along the $x$ axis, respectively. The $k$ and $c$ values can be obtained from the block's motion (computed using equation 16) that best fits the experiments of spring-block case reported in Subsection 3.2. The obtained parameters are: $k=823 \mathrm{~N} \mathrm{~m}^{-1}$ and $c=2.14 \mathrm{~N} \mathrm{~s} \mathrm{~m}^{-1}$. A rigidity of the spring $k=823 \pm 24 \mathrm{~N} \mathrm{~m}^{-1}$ was also confirmed by tensile tests and analytical determination. A major error, estimated as $20 \%$, was found in the determination of the damping coefficient c. However, a sensitivity analysis of the solution of equation 16 reveals that no large differences in the block positions are found when $\mathrm{c}$ is selected within the error range $c=2.14 \pm 0.5 \mathrm{~N} \mathrm{~s} \mathrm{~m}^{-1}$. Notice that this damping coefficient is an average equivalent damping coefficient that attempts to describe other physical phenomena like structural damping and friction.

\subsection{Modeling the experiments}

This section reports the numerical analysis of the two experimental cases reported in Section 3 , including the experimental measurements. The computational domain is discretized using 200000 cells (400 cells in $x$ and 500 in $z$ with refinement near the water/air interface). A mesh sensitivity study, equivalent to those reported in Section 2.6, was carried out in the case of the spring-damper block in air (not presented here) showing almost indistinguishable results for $\beta=l / h_{x}>50$. Following this, computations presented in the rest of the paper were performed with $\beta=100$. Slip boundary conditions are imposed along all walls, including the top and bottom of the tank. A constant time step of $0.001 \mathrm{~s}$ is adopted for the simulations. The material properties used are $\rho_{\text {water }}=998.2 \mathrm{~kg} \mathrm{~m}^{-3}, \mu_{\text {water }}=0.001 \mathrm{~Pa} \mathrm{~s}, \rho_{\text {air }}=1.225 \mathrm{~kg} \mathrm{~m}^{-3}$, and $\mu_{\text {air }}=0.000018 \mathrm{~Pa} \mathrm{~s}$ for water and air, respectively.

The material properties of the block are $\rho_{\text {solid }}=337.8 \mathrm{~kg} \mathrm{~m}^{-3}$ and $\mu_{\text {solid }}=5.10^{7} \mathrm{~Pa} \mathrm{~s}$, a value sufficient to satisfy the stiffness criterion $C_{t o t}<0.1 \%$ (see Section 2). The system is subjected to gravity action and the external horizontal acceleration promoted by the imposed motion. This horizontal acceleration is taken from the imposed experimental values as $\ddot{x}_{t a b l e}=A \omega^{2} \sin (\omega . t)$, i.e. $A_{\text {ext }}=\ddot{x}_{\text {table }}$ in equation 10 in $\Omega_{F} \cup \Omega_{S}$. The imposed amplitude $A=5 \mathrm{~mm}$ at different frequencies ranging from $1 \mathrm{~Hz}$ to $6 \mathrm{~Hz}$ are studied. The cases analysed are reported in the following subsections.

\section{Spring-block system}

The analysis for the spring-block system in the empty tank is presented in this section. The mechanical parameters $k$ and $c$ are obtained from this situation. It should be noted that $k$ identifies the existing spring, while the damping factor $c$ basically represents the structural damping and takes into account the global friction effects due to the mechanism used. As described in Section 3.1, parameters $k=823 \mathrm{~N} \mathrm{~m}^{-1}$ and $c=2.14 \mathrm{~N} \mathrm{~s} \mathrm{~m}^{-1}$ are obtained by adjusting the analytical (solution computed from equation 16) to the experimental data via a least squares procedure. Simulations are performed using such values with the aim of assessing the numerical behaviour of the proposed formulation. Numerical and experimental responses for the block motion are reported in Fig. 5 . From these plots, maximum amplitudes for the block motion and phases related to the imposed motion (table motion) are obtained. The results for all the frequencies analysed are summarized in Figs 6(a) and 6(b), respectively. Fig. 6 also includes the analytical values computed with the adopted mechanical parameters. It is remarkable that the numerical responses satisfactorily match the experiments, with the normalized root-mean-square deviation computed as $4 \%$ for amplitude and phase. The effect of varying $k$ and $c$ within the range of their errors declared in Subsection 3.1 is not reported in the present work. Nevertheless, it could be mentioned that the normalized 
root-mean-square deviation is $7 \%$ for amplitude and phase when a $c=3 \mathrm{Nsm}^{-1}$ is used. Moreover, the natural frequency of the system can be determined from Fig. 6. It is near $f_{n}=4.18 \mathrm{~Hz}$ as it could be determined analytically by $f_{n}^{2}=k / m=823 \mathrm{~N} \mathrm{~m}^{-1} / 1,193 \mathrm{Kg}$. Finally, stream lines snapshots captured at different instants of the numerical analysis for the case at $f=4 \mathrm{~Hz}$ are shown in Fig. 7, denoting the translation of the block and a disordered flow in the air. The calibrated mechanical model will be used to simulate the confined and immersed spring-block systems (i.e., cases 2 and 3$)$.

\section{Spring-block system immersed}

In this case, the spring-block system is fully immersed in the tank filled up to $h=400 \mathrm{~mm}$ and the free-surface evolves during the analysis. Figure 8 depicts the numerical and the experimental free surface evolution registered at locations S1, S2, S3, and S4 (see Fig. 3(b)). The signal reported by $\mathrm{S} 5$ is the imposed motion. This figure illustrates the physical behaviour of the free surface at the control points. The water level evolution for the reported frequencies is practically coincident at locations S1 and S2, as well as those at location S3 and S4. Moreover, the signals reported at S1 and S4 have a half-period phase shift between them. The same behaviour is observed for the free surface evolutions at S2 and S3. The numerical responses for the free surface describe these trends, but such numerical predictions lose accuracy at $1.7 \mathrm{~Hz}$. This may be due to the strong flow pattern that evolves. Fig. 9 plots the computed block motion together with the experimental data. The imposed motion is also included to denote the phases. In spite of the differences reported for the numerical predictions of the free surface, a very good agreement can be observed between numerical and experimental results for the block motion. Maximum amplitudes of the block motion and phases are reported in Fig. 10 for the different imposed frequencies. A satisfactory correspondence can be seen between numerical responses and experimental data. The maximum errors in amplitude and phases are obtained near resonance conditions and they are bounded by $11 \%$ and $7 \%$, respectively. The graphs show that the resonance occurs at the frequency $f_{n}=1.4 \mathrm{~Hz}$, which is the natural frequency of the system without the block $\left(f_{n}=(g /(4 \pi L) \tanh (\pi h / L))^{0,5}\right)$. At this frequency the block amplitude is the highest and the phase difference is approximately $\pi / 2$. A negative phase lag is also apparent at the frequency of $1.0 \mathrm{~Hz}$. The interaction between the block and the free surface causes the block to move forwards with respect to the tank at some frequencies. Stream lines at different instants of the analysis are presented in Fig. 11, which shows that the block translation is properly captured as well as the free surface conditions. Vortex generation is also observed in water due to the cyclic motion of the block.

\section{Examples of rotational cases}

A few engineering devices such as the oscillating wave surge converters (e.g. the Wave Oyster or the Wave Roller systems described by Folley, Whittaker, \& Hoff, 2007), involves a solid in rotational motion around an axis. Therefore, there is a need for the simulation of this kind of wave-flow /structure interactions. Two examples are shown in this section to illustrate the capability of the method to deal with rotational motions.

In the first one, a rectangular body is forced to move in rotation around an axis located in the bottom part of the solid. The methodology used to achieve this rotation was explained in 2.3. The surrounding fluid is only air. The fluid viscosity within the solid area is $\mu_{\text {solid }}=5.10^{7} \mathrm{~Pa} \mathrm{~s}$ and its density is $500 \mathrm{~kg} \mathrm{~m}^{-3}$. The body is subjected to a restoring and a damping moment imposed as body forces (2.3). Gravity is set to zero. The solid body initial inclination is $45^{\circ}$ compared to its equilibrium position which is here vertical. Figure 12 shows snapshots of the solid position and streamlines at two different times. The center of rotation is clearly visible in the bottom part of the solid. Around the rotation axis, flow streamlines are strictly circular within the solid area. 
Figure 12-b shows the flow after approximately one half of the rotation cycle. Recirculation and flow vorticity are generated in the air flow by the solid motion. In this case, the air influence on the solid is weak and the solid motion is very close to the analytical solution of the classical oscillator without surrounding fluid (Ducassou, 2016).

The second example shows the application of the penalty numerical method to the case of a seabed-mounted bottom-hinged wave energy converter (WEC) described also by Gomes, Lopes, Henriques, Gato, and Falcao (2015). The device is forced to move rotationally under the action of regular waves. The power take-off is a hydraulic damper. The following dimensions are considered in the simulation: water depth $h=200 \mathrm{~mm}$, device/wave source distance $l=3500 \mathrm{~mm}=17.5 h$, device height $D=140 \mathrm{~mm}$ and width $e=30 \mathrm{~mm}$ and rotation center position $Z_{p}=30 \mathrm{~mm}$. Flap solid body behaviour is achieved using a viscosity value of $5.10^{7} \mathrm{~Pa} \mathrm{~s}$.

The regular wave is generated through a source term added in the mass conservation equation following the method described in Lin and Liu (1999) associated with a fifth order Stokes theory. With this approach, waves are propagated in both directions. In the cases presented here, wave period is $T_{w}=1.5 \mathrm{~s}$, wave height $H_{w}=20 \mathrm{~mm}=0.1 \mathrm{~h}$ and wave length $L_{w}=2060 \mathrm{~mm}=10.3 \mathrm{~h}$.

Two configurations are studied in this paper: the oscillating flap is in open water (i.e., regular wave trains are described) and the upstream limit of the domain is a reflective wall (i.e., standing waves are generated). The first case is sketched in Fig. 13. In order to avoid multiple interference after reflection at the boundaries, two dissipative zones made up of a porous medium (as proposed by Khadra, Angot, Parneix, and Caltagirone (2000)) are imposed at both lateral limits of the domain to mimic open water cases, while waves are only damped at the upstream (left) limit in the case of a reflective wall (i.e., no dissipation zone is used at the right limit of the domain).

The mesh is made up of 1350 cells in $x$ and 250 cells in $z$. The initial time step is $0.1 \mathrm{~s}$ to allow the computation to start from a null velocity field. After the first instants of the analysis a Courant number of 0.2 is used for the remainder of the simulation.

To run the cases including waves,two parameters need to be characterized in order to optimise the system's efficiency: the flap density and the rotational damping coefficient $c_{r}$. Energy transfer is minimized when the system works near resonance conditions, i.e., the natural oscillating frequency of the device in water needs to be close to the wave frequency. Hence, a parametric study was conducted (not all results are shown) to determine the density value that ensures such condition. The flap was subjected to free vibration by inducing an initial shift from its equilibrium position. Simulations were performed with different density values (from $50 \mathrm{~kg} \mathrm{~m}^{-3}$ to $900 \mathrm{~kg} \mathrm{~m}^{-3}$ ), without and with free surface. Notice that the added mass and buoyancy effects play a relevant role in such computations (e.g., as was demonstrated by Costarelli et al., 2016).

Figure 14 presents snapshots of a free vibration analysis without free surface. This figure shows the formation of dipole vortices stressing the complexity of the flow and the interest of the NavierStokes simulations in comparison with more classical potential flow models. From these simulations, a density of $200 \mathrm{~kg} \mathrm{~m}^{-3}$ ensures that the natural frequency of the WEC is close to the wave period (i.e., $1.74 \mathrm{~s}$ for the device against $1.5 \mathrm{~s}$ for the waves, in the present analysis). The latter value is chosen in the present the study.

The simulation of the free vibration analysis including free surface effects (Fig. 15) stress the importance of the latter on the evolution of the solid body. In this case, conversely to the preceding one, the solid motion is immediately damped and the WEC directly goes to its equilibrium position without oscillating.

Following the mechanical characterization of the system, a feasible rotational damping coefficient needs to be determined. To this end, the simulations with waves (forced vibration) were performed using three values of such a coefficient: $c_{r}=0 \mathrm{~N} \mathrm{~m} \mathrm{~s}, c_{r}=3 \mathrm{~N} \mathrm{~m} \mathrm{~s}$ and $c_{r}=30 \mathrm{~N} \mathrm{~m} \mathrm{~s}$. Figure 16 shows the wave-interaction effect on the device after the initial transient regime is completed, for the $c_{r}=0 \mathrm{~N} \mathrm{~m} \mathrm{s.} \mathrm{A} \mathrm{complex} \mathrm{pattern} \mathrm{of} \mathrm{streamlines} \mathrm{develops} \mathrm{as} \mathrm{waves} \mathrm{propagate} \mathrm{showing} \mathrm{the}$ interaction with the WEC. As in Fig. 12, the rotation center is clearly visible by the concentric circles of streamlines at the bottom of the flap. Streamlines are obviously circular curves anywhere 
in the solid area, a fact that confirms the ability of the rigidity criterion to promote solid behaviour while rotational motion occurs. A complex vortex structure evolves near the tip of the flap over time. There are also some vortices created close to the bottom of the domain due to the unavoidable void between the moving flap and its attachment. It is interesting to note that many numerical methods previously used to study wave energy converters do not take into account viscous drag and vortex shedding, or such effects are considered by adding explicit terms to be parametrized. Here the method allows an implicit handling of vortex shedding which may be an important cause of dissipation for oscillating wave surge converters. To quantify the obtained overall numerical results, the system's efficiency $\eta$, defined as: $\eta=8 \int_{0}^{T} c_{r} \dot{\theta}^{2} d t /\left(\rho g H_{w}^{2} L_{w}\right)$, has been computed. It also helps to provide a qualitative comparison with the studies reported by Gomes et al. (2015).

In open water, the obtained mean amplitude of the oscillating flap are $12^{\circ}, 3^{\circ}$ and $0.1^{\circ}$ for $c_{r}=0$ $\mathrm{N} \mathrm{m} \mathrm{s}, c_{r}=3 \mathrm{~N} \mathrm{~m} \mathrm{~s}$ and $c_{r}=30 \mathrm{~N} \mathrm{~m} \mathrm{~s}$, respectively. Accordingly, the efficiency is $\eta=20.5 \%$ and $\eta=1.3 \%$ for $c_{r}=3 \mathrm{~N} \mathrm{~m} \mathrm{~s}$ and $c_{r}=30 \mathrm{~N} \mathrm{~m} \mathrm{~s}$, respectively (notice that null efficiency is computed for $c_{r}=0 \mathrm{~N} \mathrm{~m} \mathrm{~s}$ ).

The situation changes drastically when a reflective wall at the right boundary is used. In such a case, a standing wave is generated and the relative WEC position to the wall plays a crucial role. For the flap located at an antinode of the standing wave and $1570 \mathrm{~mm}$ apart from the right of the domain, the obtained mean amplitudes of the oscillating flap are $18^{\circ}, 6^{\circ}$ and $0.3^{\circ}$ for $c_{r}=0 \mathrm{~N} \mathrm{~m}$ $\mathrm{s}, c_{r}=3 \mathrm{~N} \mathrm{~m} \mathrm{~s}$ and $c_{r}=30 \mathrm{~N} \mathrm{~m} \mathrm{~s}$, respectively. The maximum efficiency is $\eta=59.7$ for $c_{r}=3$ $\mathrm{N} \mathrm{m}$ s. For the flap positioned close to a node of the standing wave, the efficiency reduces to $5 \%$ using the same model parameters.

Finally, the obtained efficiency trends are close to those reported in the literature when similar wave conditions and devices are used (see Gomes et al. (2015) and references therein). This fact qualitatively assess the numerical behaviour of the proposed methodology.

\section{Concluding remarks}

In the present work a numerical strategy has been proposed to solve wave/structure interactions, including the modelling of free surface evolution and external forces acting on the solid. The methodology was developed in the well known framework of staggered finite volume techniques where the moving interfaces, that is free surface and body contour, were updated using a volume of fluid approach. The formulation proposes to enforce the rigid conditions via penalization of the fluid viscosity. Spring-damper forces acting on the solid are considered as body forces per unit mass distributed into the solid domain. Kinematic constraints are imposed on the solidified area by simply penalizing velocity nodes.

In addition, an experiment was also conducted to assess the capabilities of the present numerical strategy. Amplitudes of the block position and their phases, and the free surface evolution have been satisfactorily validated by contrasting the numerical responses with the experimental data using different imposed motion conditions.

Finally, numerical examples have been given to illustrate the ability of the method to deal with rotational solid motion in interaction with waves. The system's efficiency, chosen as an overall parameter to verify the numerical responses, has shown similar trends to those reported in the literature for the waves and mechanical conditions analysed. Future work will be the experimental validation of the model for rotational motions as well as $3 \mathrm{D}$ analyses.

\section{Funding}

The authors thank the support given by the French government, the ECOS-Sud committee, and the Chilean Comisión Nacional de Investigación Científica y Tecnolgica (CONICYT): EMA- 
COP, ECOS-CONICYT C12E01, and CONICYT-FONDECYT 1130278, the Scientific Research Projects Management Department of the Vice Presidency of Research, Development and Innovation (DICYT-VRID) of the Universidad de Santiago de Chile, and Project Basal USA1498. 


\section{References}

Abadie, S., Caltagirone, J., \& Watremez, P. (1998). Splash-up generation in a plunging breaker. Comptes Rendus de l'Academie des Sciences Series IIB Mechanics Physics Astronomy, 326 (9), 553-559.

Abadie, S., Morichon, D., Grilli, S., \& Glockner, S. (2010). Numerical simulation of waves generated by landslides using a multiple-fluid navier-stokes model. Coastal Engineering, 57(9), 779794.

Altomare, C., Crespo, A., Rodgers, B., Dominguez, J., Gironella, X., \& Gomez-Gesteira, M. (2014). Numerical modelling of armour block sea breakwater with smoothed particle hydrodynamics. Computers and Structures, 130, 34-45.

Angot, P., Bruneau, C.-H., \& Fabrie, P. (1999). A penalization method to take into account obstacles in incompressible viscous flows. Numerische Mathematik, 81(4), 497-520.

Borgarino, B., Babarit, A., \& Ferrant, P. (2012). An implementation of the fast multipole algorithm for wave interaction problems on sparse array of floating bodies. J. Eng. Math., 77, 51-68.

Caltagirone, J.-P., \& Vincent, S. (2001). Sur une méthode de pénalisation tensorielle pour la résolution des équations de navier-stokes. Comptes Rendus de l'Académie des Sciences-Series IIB-Mechanics, 329(8), 607-613.

Costarelli, S. D., Garelli, L., Cruchaga, M. A., Storti, M. A., Ausensi, R., \& Idelsohn, S. R. (2016). An embedded strategy for the analysis of fluid structure interaction problems. Computer Methods in Applied Mechanics and Engineering, 300, 106-128.

Cruchaga, Celentano, D., \& Tezduyar, T. (2007). A numerical model based on the mixed interfacetracking/interface-capturing technique (mitict) for flows with fluid-solid and fluid-fluid interfaces. International journal for numerical methods in fluids, 54(6-8), 1021-1030.

Cruchaga, Löhner, R., \& Celentano, D. (2012). Experimental and numerical analysis of a sphere falling into a viscous fluid. International Journal for Numerical Methods in Fluids, 69(9), $1496-1521$.

Cruchaga, Muñoz, C. M., \& Celentano, D. J. (2008). Simulation and experimental validation of the motion of immersed rigid bodies in viscous flows. Computer Methods in Applied Mechanics and Engineering, 197 (33), 2823-2835.

Donea, R., Giuliani, S., \& Halleux, J. (1982). An arbitrary lagrangian-eulerian finite element method for transient dynamic fluid-structure interactions. Computer methods in applied mechanics and engineering, 33(1), 689-723.

Ducassou, B. (2016). Modélisation de l'interaction entre un fluide à surface libre et un solide rigide soumis à des efforts extérieur par une méthode de pénalisation (doctoral dissertation). Université de Pau et des Pays de l'Adour, France. (In French)

Folley, M., Whittaker, T., \& Hoff, J. V. (2007). The design of small seabed-mounted bottom-hinged wave energy converters. In Proceedings of the 7th european wave and tidal energy conference.

Fortin, M., \& Glowinski, R. (1982). Méthodes de lagrangien augmenté: applications à la résolution numérique de problèmes aux limites. Dunod.

Gingold, R. A., \& Monaghan, J. J. (1977). Smoothed particle hydrodynamics: theory and application to non-spherical stars. Monthly notices of the royal astronomical society, 181(3), $375-389$.

Glowinski, R., Pan, T., Hesla, T., Joseph, D., \& Periaux, J. (2001). A fictitious domain approach to the direct numerical simulation of incompressible viscous flow past moving rigid bodies: application to particulate flow. Journal of Computational Physics, 169(2), 363-426.

Gomes, R., Lopes, M., Henriques, J., Gato, L., \& Falcao, A. (2015). The dynamics and power extraction of bottom-hinged plate wave energy converters in regular and irregular waves. Ocean Engineering, 96, 86-99.

Groenenboom, P. H., \& Cartwright, B. K. (2010). Hydrodynamics and fluid-structure interaction by coupled sph-fe method. Journal of Hydraulic Research, 48 (sup1), 61-73. 
Harlow, F. H., Welch, J. E., et al. (1965). Numerical calculation of time-dependent viscous incompressible flow of fluid with free surface. Physics of fluids, 8(12), 2182.

Hirt, C. W., \& Nichols, B. D. (1981). Volume of fluid (vof) method for the dynamics of free boundaries. Journal of computational physics, 39(1), 201-225.

Huang, Z., He, F., \& Zhang, W. (2014). A floating box-type breakwater with slotted barriers. Journal of Hydraulic Research, 52, 720-727.

Kassiotis, C., Ibrahimbegovic, A., \& Matthies, H. (2010). Partitioned solution to fluid-structure interaction problem in application to free-surface flows. European Journal of MechanicsB/Fluids, 29(6), 510-521.

Khadra, K., Angot, P., Parneix, S., \& Caltagirone, J.-P. (2000). Fictitious domain approach for numerical modelling of navier-stokes equations. International Journal for Numerical Methods in Fluids, 34 (8), 651-684.

Koftis, T., Prinos, P., \& Koutandos, E. (2006). 2d-v hydrodynamics of wave-floating breakwater interaction. Journal of Hydraulic Research, 44, 451-469.

Lin, P., \& Liu, P. (1999). Internal wave-maker for navier-stokes equations models. Journal of Waterways, Port, Coastal and Ocean Engineering, 125, 207-215.

Nuñez, J. (2015). Análisis experimental y numérico del comportamiento en vibraciones de un sistema fluido-bloque rígido en distintas condiciones de inmersión (master dissertation). Universidad de Santiago de Chile (USACH), Chile. (In Spanish)

Patankar, S. (1980). Numerical heat transfer and fluid flow. CRC Press.

Peskin, C. S. (1972). Flow patterns around heart valves: a numerical method. Journal of computational physics, 10(2), 252-271.

Peskin, C. S. (2002). The immersed boundary method. Acta numerica, 11, 479-517.

Randrianarivelo, T., Pianet, G., Vincent, S., \& Caltagirone, J. (2005). Numerical modelling of solid particles motion using a new penalty method. Int. J. Numer. Meth. Fluids, 47, 1245-1251.

Renzi, E., Abdolali, A., Bellotti, G., \& Dias, F. (2014). Wave-power absorption from a finite array of oscillating wave surge converters. Renewable Energy, 63, 55-68.

Ritz, J., \& Caltagirone, J. (1999). A numerical continuous model for the hydrodynamics of fluid particle systems. International Journal for Numerical Methods in Fluids, 30(8), 1067-1090.

Rogers, B. D., Dalrymple, R. A., \& Stansby, P. K. (2010). Simulation of caisson breakwater movement using 2-d sph. Journal of Hydraulic Research, 48, 135-141.

Sagaut, P., Troff, B., Lê, T., \& Loc, T. P. (1996). Large eddy simulation of turbulent flow past a backward facing step with a new mixed scale sgs model. In Computation of three-dimensional complex flows (pp. 271-277). Springer.

Sarthou, A., Vincent, S., Caltagirone, J., \& Angot, P. (2008). Eulerian-lagrangian grid coupling and penalty methods for the simulation of multiphase flows interacting with complex objects. International Journal for Numerical Methods in Fluids, 56(8), 1093-1099.

Ten Cate, A., Nieuwstad, C., Derksen, J., \& Van den Akker, H. (2002). Particle imaging velocimetry experiments and lattice-boltzmann simulations on a single sphere settling under gravity. Physics of Fluids (1994-present), 14 (11), 4012-4025.

Veeramani, C., Minev, P. D., \& Nandakumar, K. (2007). A fictitious domain formulation for flows with rigid particles: A non-lagrange multiplier version. Journal of Computational Physics, 224 (2), 867-879.

Vincent, S., Randrianarivelo, T., Pianet, G., \& Caltagirone, J. (2007). Local penalty methods for flows interacting with moving solids at high reynolds numbers. Computers and Fluids, 36, 902-913.

Yu. (2005). A dlm/fd method for fluid/flexible-body interactions. Journal of computational physics, $207(1), 1-27$.

Yu, Y.-H., \& Li, Y. (2013). Reynolds-averaged navierstokes simulation of the heave performance of a two-body floating-point absorber wave energy system. Computers and Fluids, 73, $104-$ 114. 
Zhao, X., Ye, Z., Fu, Y., \& Cao, F. (2014). A cip-based numerical simulation of freak wave impact on a floating body. Ocean Engineering, 87, $50-63$.

Tables and figures

Table 1: Dimensions of the experimental system

\begin{tabular}{|c|c|c|}
\hline Tank height & $L_{1}$ & $500 \pm 1 \mathrm{~mm}$ \\
\hline Tank width & $L$ & $400 \pm 1 \mathrm{~mm}$ \\
\hline Tank depth & $E$ & $200 \pm 1 \mathrm{~mm}$ \\
\hline Block height & $h$ & $200 \pm 1 \mathrm{~mm}$ \\
\hline Block width & $l$ & $90 \pm 1 \mathrm{~mm}$ \\
\hline Block depth & $e$ & $20 \pm 1 \mathrm{~mm}$ \\
\hline Block mass & $m$ & $1.192 \pm 0.001 \mathrm{~kg}$ \\
\hline
\end{tabular}

$\Gamma$

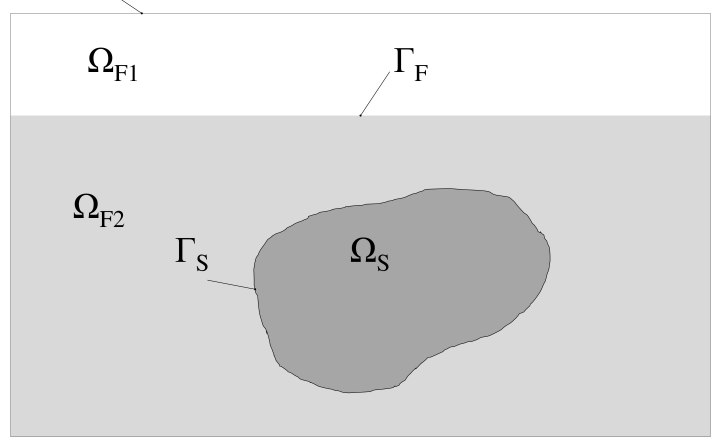

Figure 1: Sketch of the studied case 
(a)

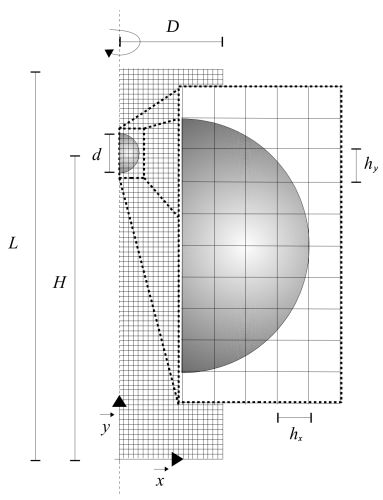

(b)

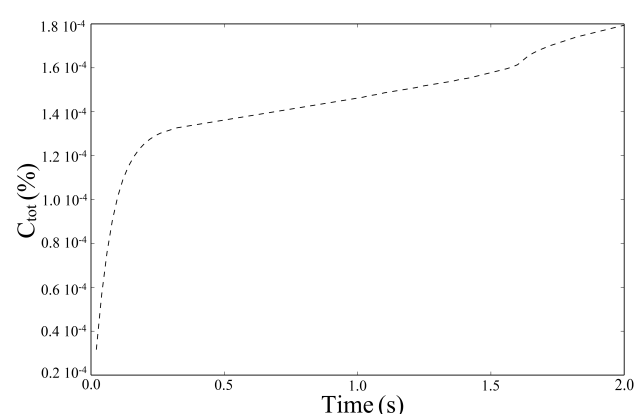

(c)

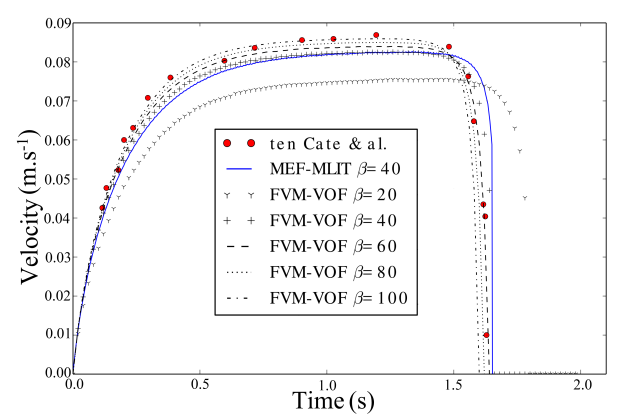

Figure 2: Sphere setting case: (a) computation grid; (b) evolution of accumulated deformation; and (c) mesh size convergence.

(a)

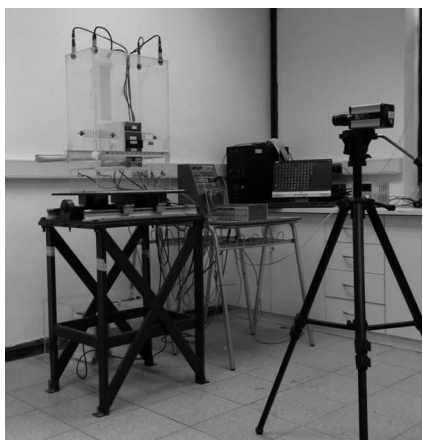

(b)

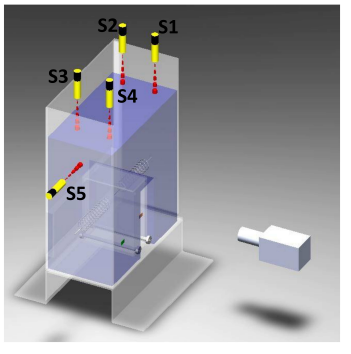

(c)

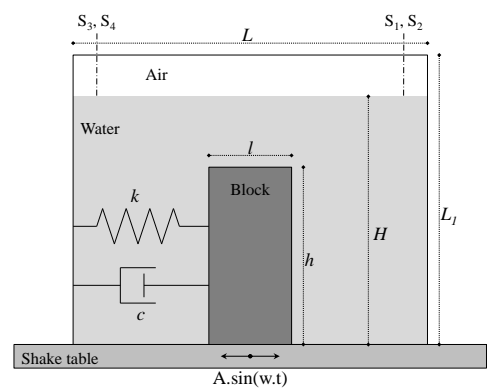

Figure 3: Experimental setup: (a) view of the laboratory, (b) 3D plot including sensor positions and (c) sketch of the problem studied.

(a)

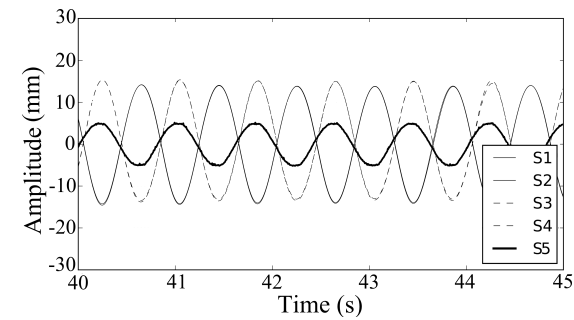

(b)

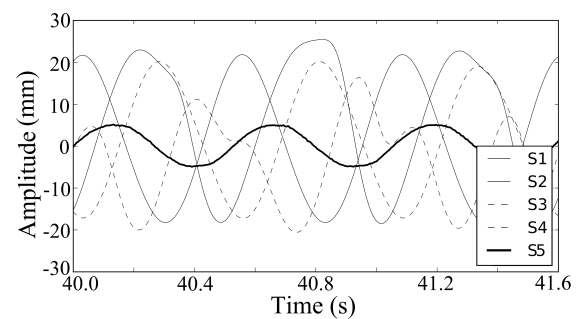

Figure 4: Experimental free surface time evolution: (a) $2 \mathrm{D}$ behaviour at $\mathrm{f}=1.25 \mathrm{~Hz}$ and (b) $3 \mathrm{D}$ behaviour at $\mathrm{f}=1.9 \mathrm{~Hz}$. 
(a)

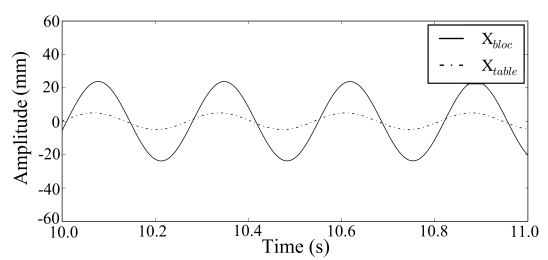

(c)

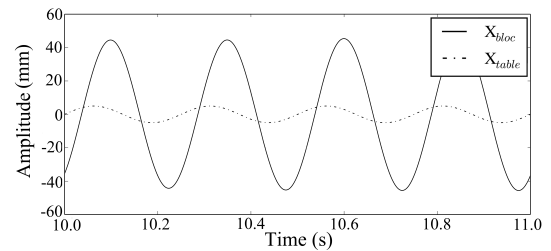

(e)

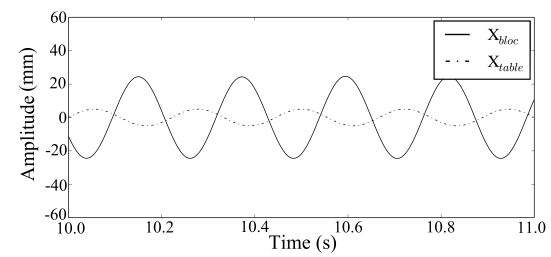

(b)

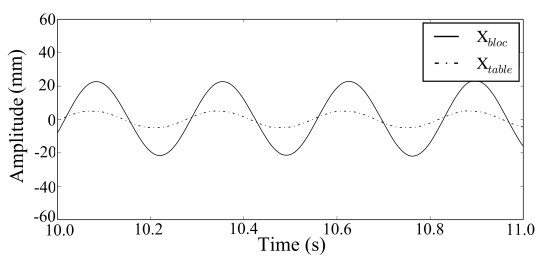

(d)

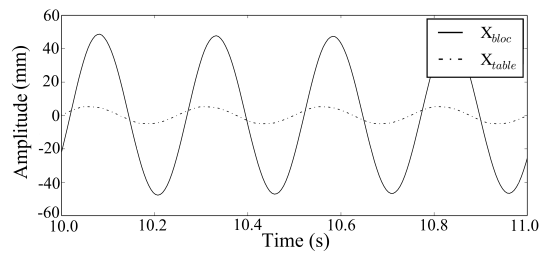

(f)

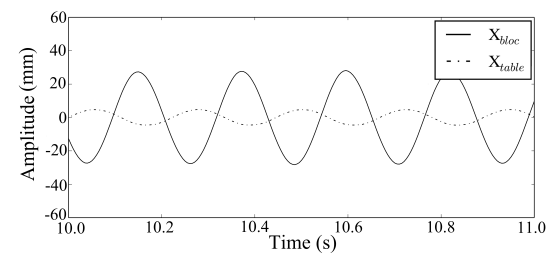

Figure 5: Spring-block in air only. Numerical (a, c, e) and experimental (b, d, f) block displacements evolution during the time-periodic regime for imposed motion of frequencies $3.7 \mathrm{~Hz}$ (a and b), 4.0 $\mathrm{Hz}$ (c and d), and $4.5 \mathrm{~Hz}$ (e and f). Shake table displacement is also included.
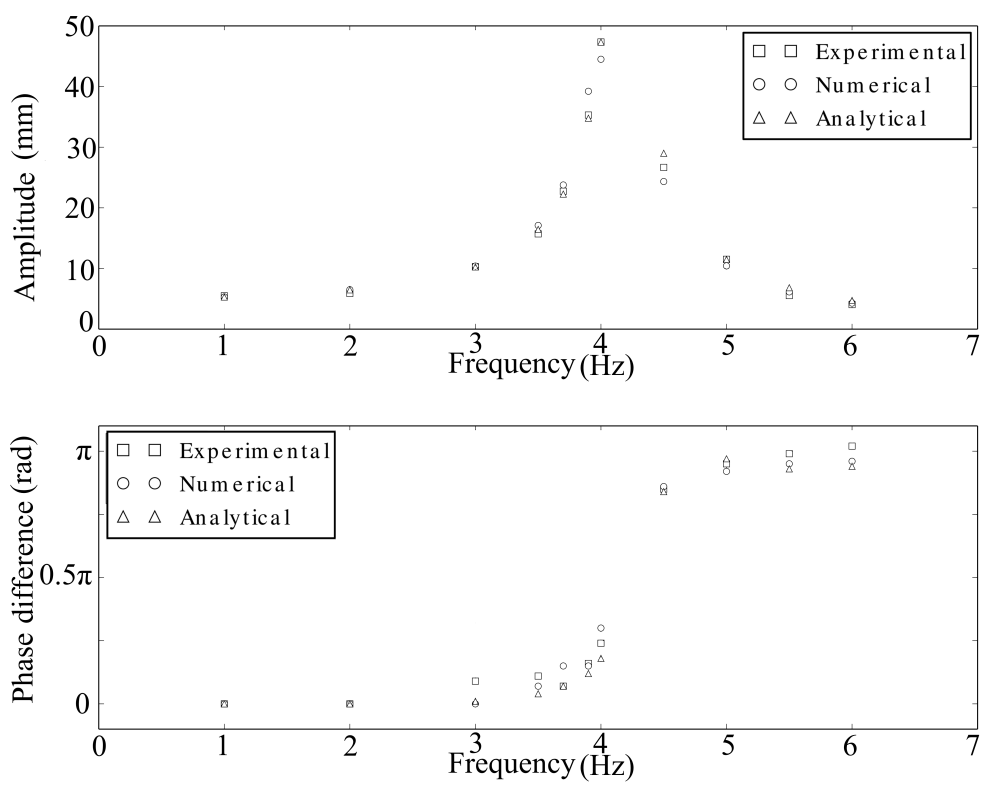

Figure 6: Spring-block in air only. Amplitude and phase diagrams. 
(a)

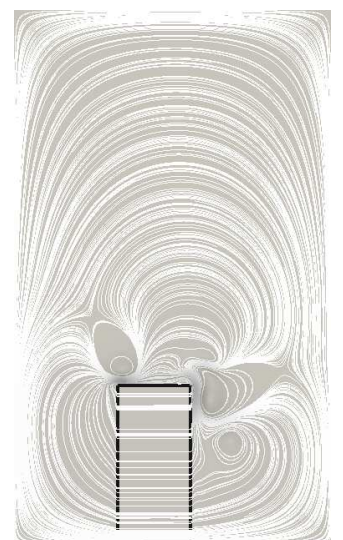

(b)

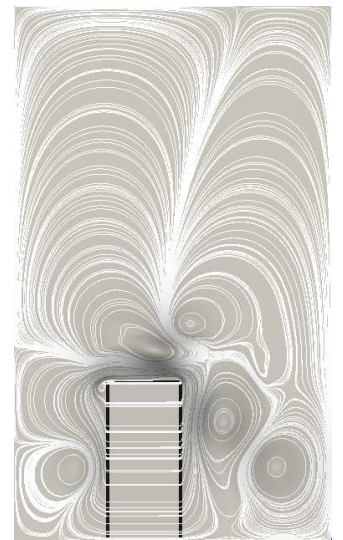

(c)

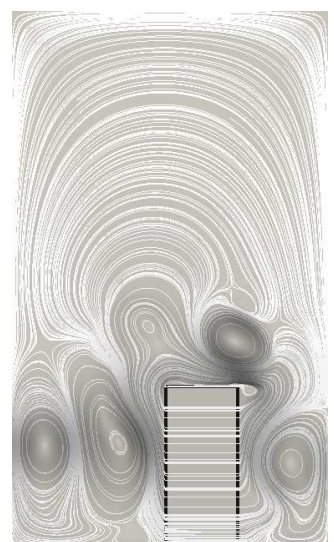

(d)

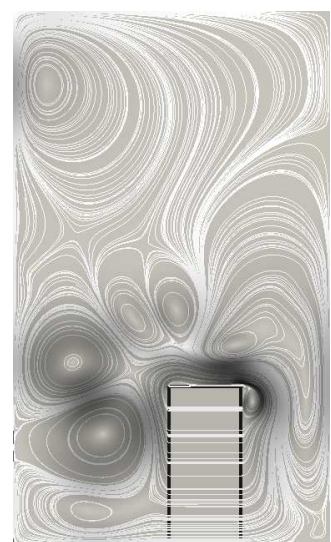

Figure 7: Spring-block in air only. Snapshots at instants $10.22 \mathrm{~s}$ (a), $10.25 \mathrm{~s}$ (b), 10.30 (c) s, and $10.32 \mathrm{~s}(\mathrm{~d})$ of the numerical simulations with imposed motion at frequency $4.0 \mathrm{~Hz}$.

(a)

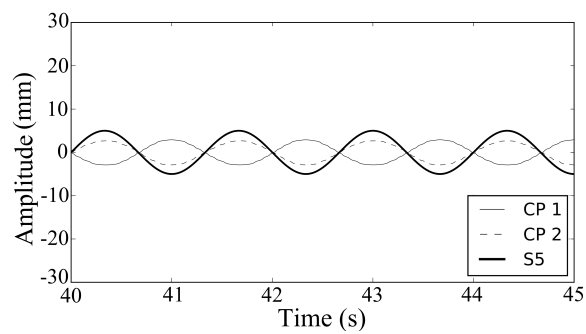

(c)

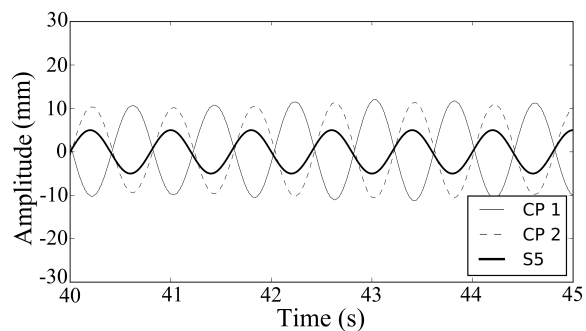

(e)

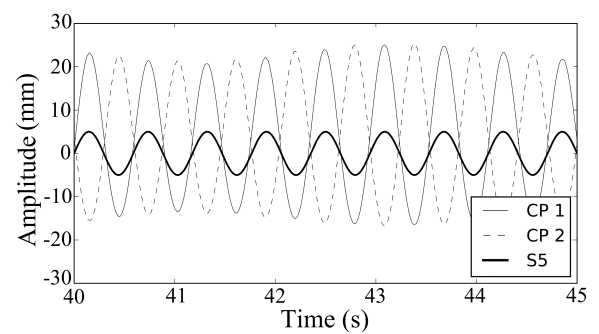

(b)

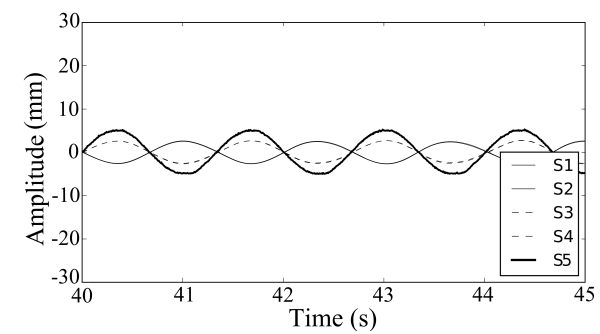

(d)

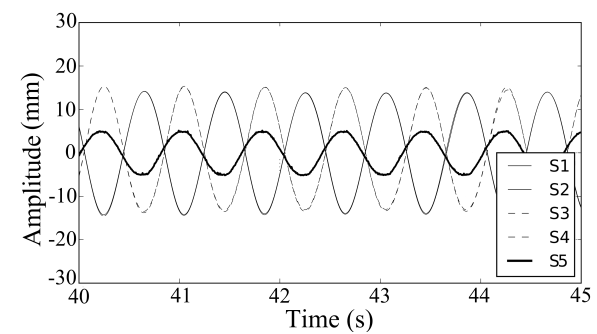

(f)

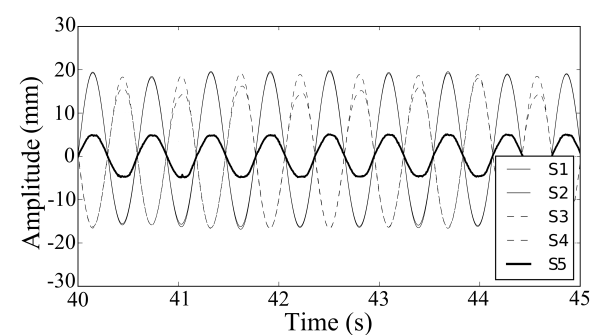

Figure 8: Spring-block in $400 \mathrm{~mm}$ of water. Numerical (a, c, e) and experimental (b, d, f) water level evolution during the time-periodic regime at imposed motions of $0.75 \mathrm{~Hz}(\mathrm{a}, \mathrm{b}), 1.25 \mathrm{~Hz}(\mathrm{c}, \mathrm{d})$ and $1.7 \mathrm{~Hz}(\mathrm{e}, \mathrm{f})$. 
(a)

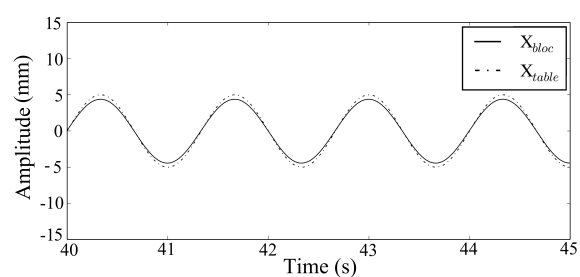

(c)

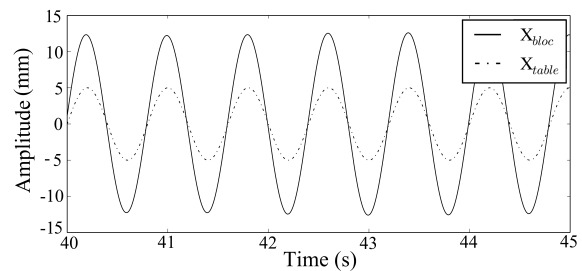

(e)

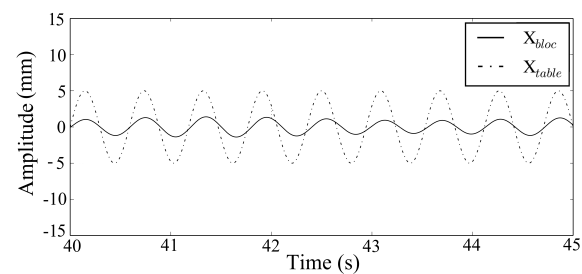

(b)

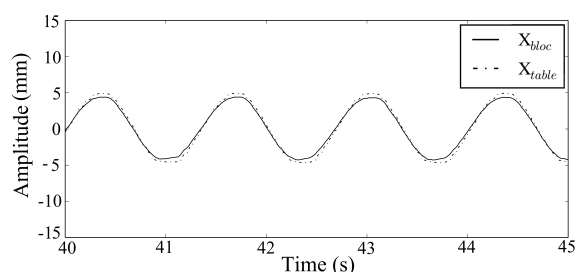

(d)

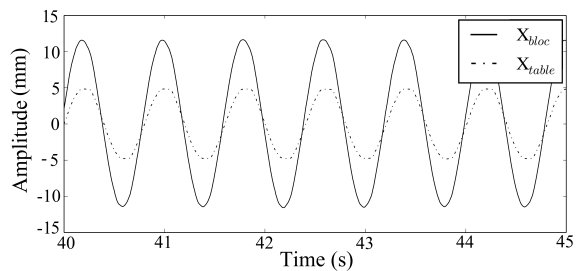

(f)

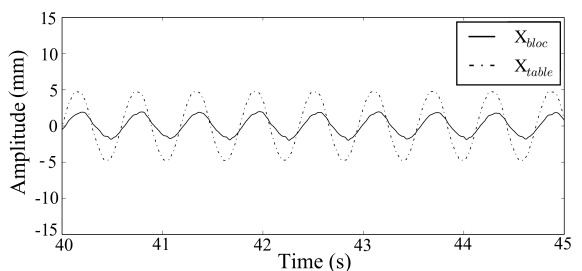

Figure 9: Spring-block in $400 \mathrm{~mm}$ of water. Numerical (a,c,e) and experimental (b,d,f) block displacements evolution during the time-periodic regime at frequencies of $0.75 \mathrm{~Hz}(\mathrm{a}, \mathrm{b}), 1.25 \mathrm{~Hz}(\mathrm{c}, \mathrm{d})$ and $1.7 \mathrm{~Hz}(\mathrm{e}, \mathrm{f})$. Shake table displacement is also included.
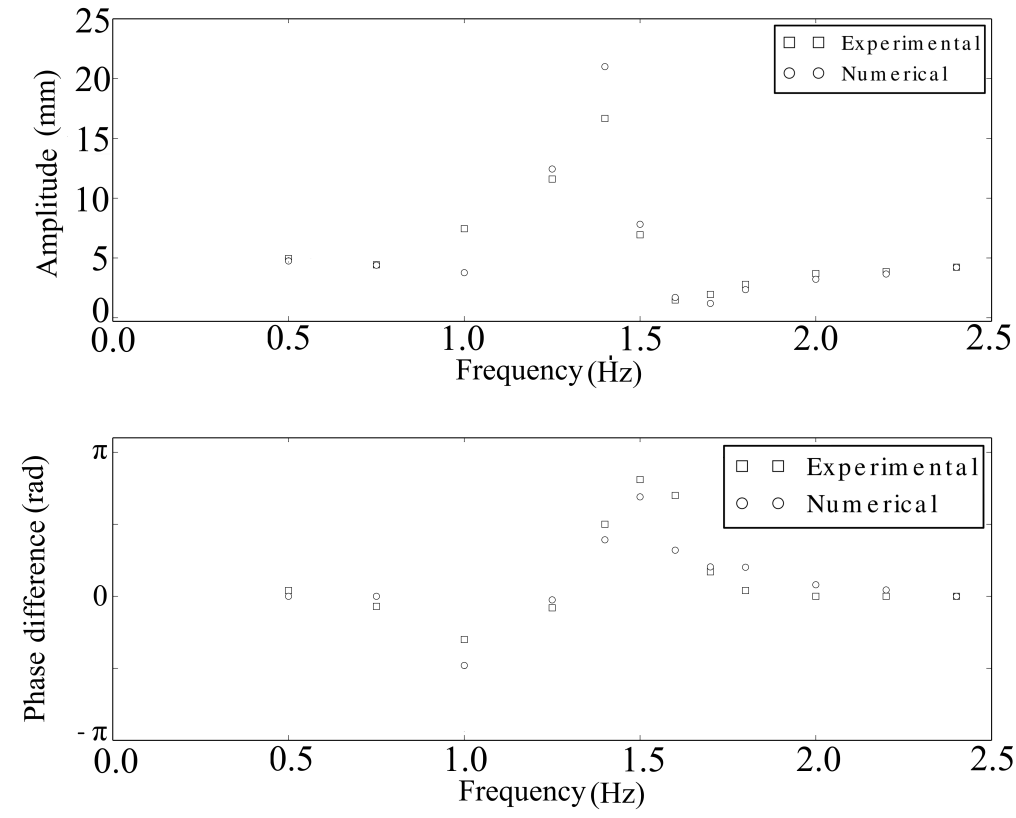

Figure 10: Spring-block in $400 \mathrm{~mm}$ of water. Amplitude and phase diagrams. 
(a)

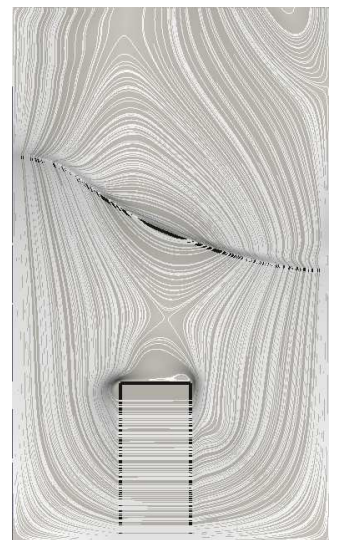

(b)

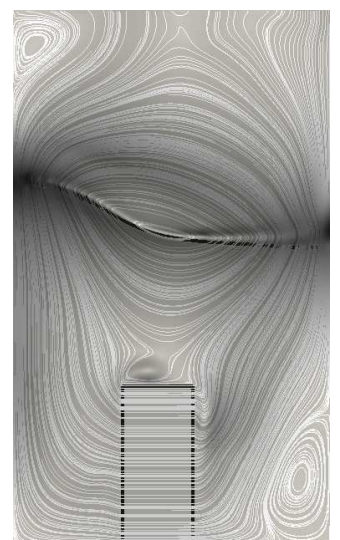

(c)

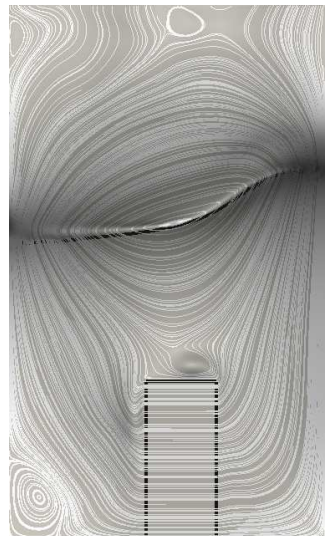

(d)

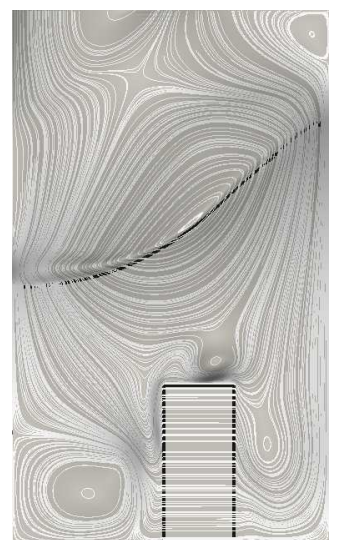

Figure 11: Spring-block in $400 \mathrm{~mm}$ of water. Snapshots of numerical simulation at $1.4 \mathrm{~Hz}$ and at $40.2 \mathrm{~s}(\mathrm{a}), 40.27 \mathrm{~s}(\mathrm{~b}), 40.48 \mathrm{~s}$ (c) and $4.55 \mathrm{~s}(\mathrm{~d})$.

(a)

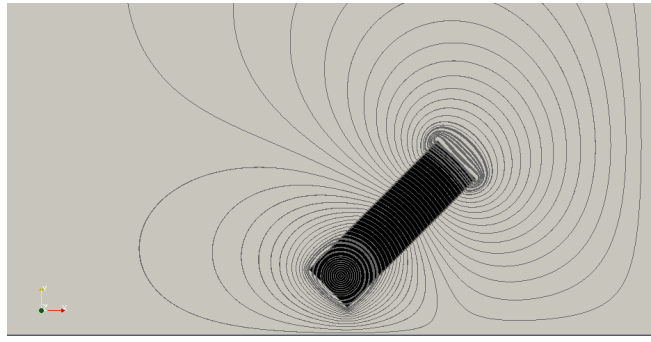

(b)

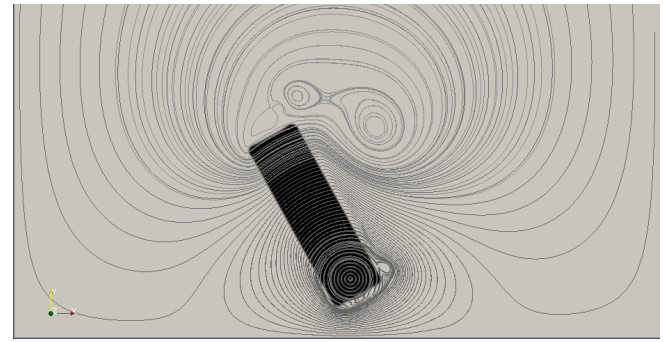

Figure 12: Rectangular solid forced to move in rotation and subjected to restoring and damping moments. Surrounding fluid is only air. Immediately after solid release (i.e. $t \approx 0$ ) (a) and after half a period of oscillation (b).

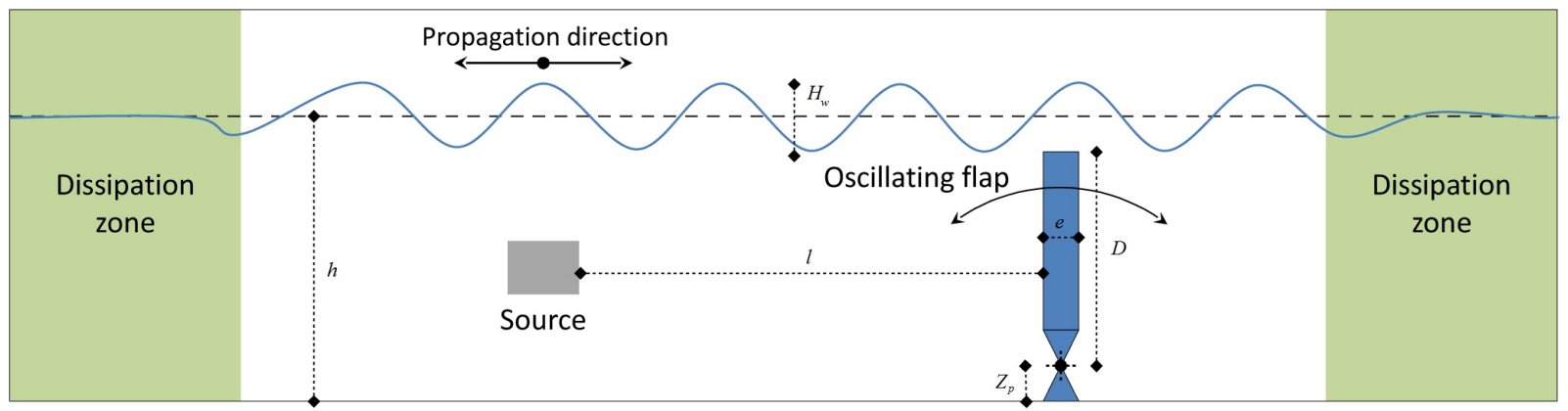

Figure 13: Sketch of the computational domain for the seabed-mounted bottom-hinged wave energy converter simulation. 
(a)

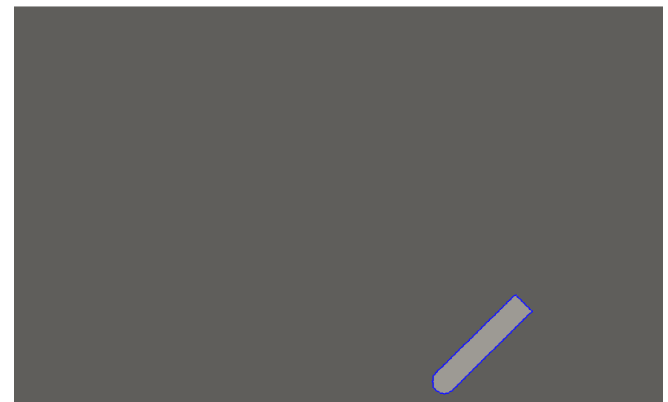

(c)

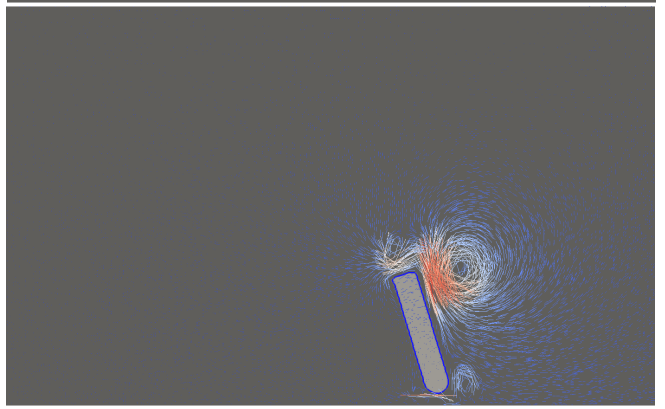

(b)

(d)
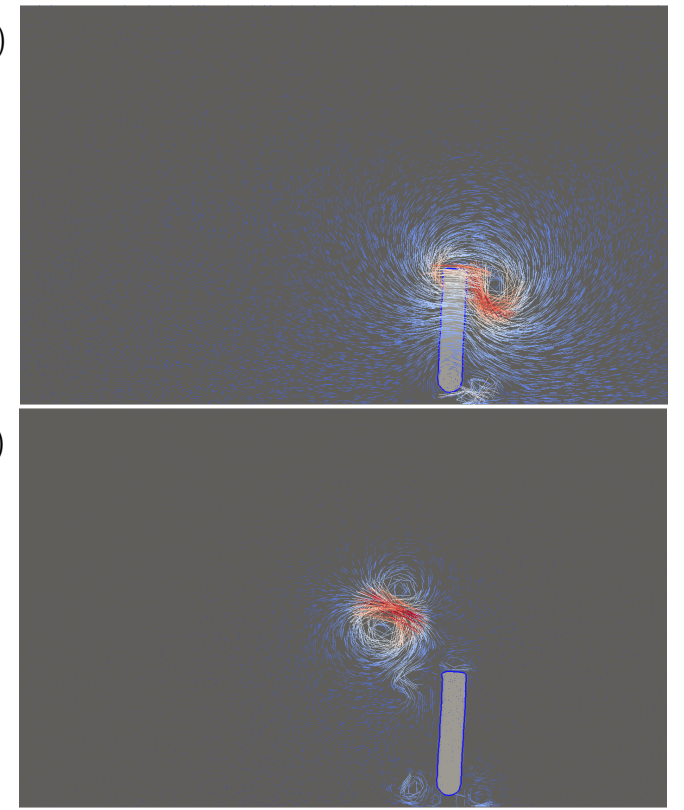

Figure 14: Simulation of the free vibration WEC motion in water without free surface at different instants: $t=0 \mathrm{~s}(\mathrm{a}), t=0.487 \mathrm{~s}(\mathrm{~b}), t=0.899 \mathrm{~s}(\mathrm{c}), t=1.778 \mathrm{~s}(\mathrm{~d})$.

(a)

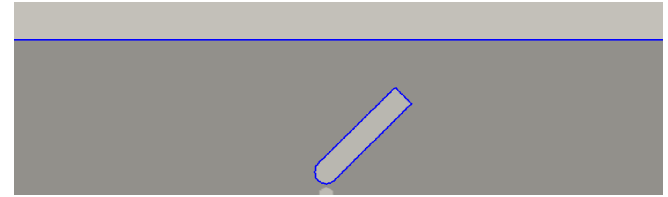

(c)

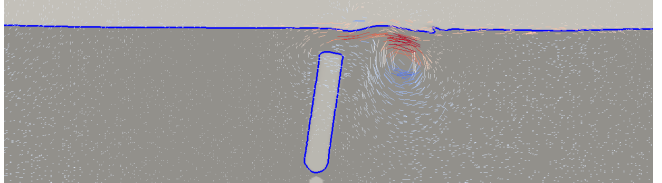

(b)

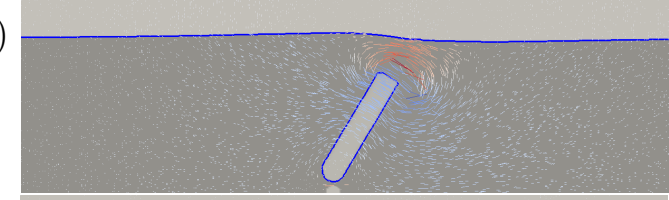

(d)

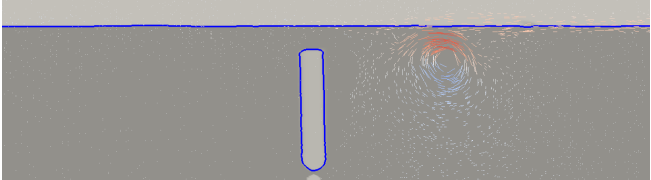

Figure 15: Simulation of the free vibration WEC motion in water with free surface at different instants: $t=0 \mathrm{~s}(\mathrm{a}), t=0.282 \mathrm{~s}(\mathrm{~b}), t=0.885 \mathrm{~s}(\mathrm{c}), t=2.439 \mathrm{~s}(\mathrm{~d})$.

(a)

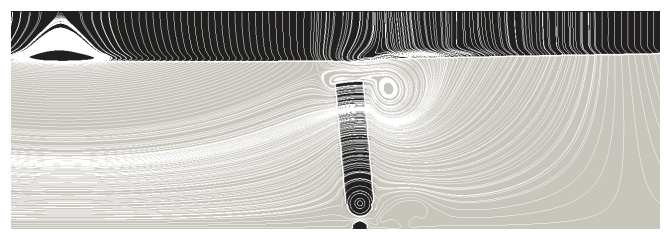

(b)

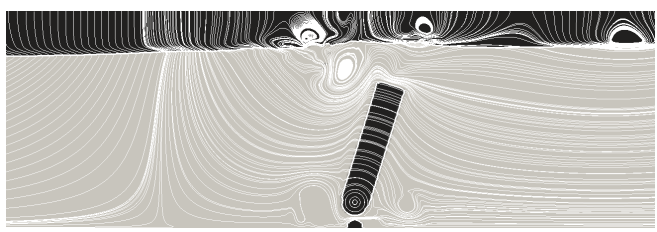

Figure 16: Simulation of the oscillating WEC in interaction with a regular wave train (open water). Snapshots of fluid interfaces and streamlines: wave crest at the left boundary (a) and wave crest above the flap (b). 